\title{
Reviewers in 2015
}

(C) The Japan Society of Brain Tumor Pathology 2015

Brain Tumor Pathology Editorial Office wishes to thank the following reviewers (non-editorial board members) for their contributions in 2015.

Hideyuki Arita, Osaka

Kanako Hatanaka, Hokkaido

Keisuke Ishizawa, Saitama

Kenta Masui, Tokyo

Hadzki Matsuda, Tochigi

Satoko Nakada, Ishikawa

Hiroshi Nishihara, Hokkaido

Masayuki Nitta, Tokyo

Sumihito Nobusawa, Gunma

Norihiko Saito, Tokyo

Tatsuo Sawada, Tokyo

Akihiko Yoshida, Tokyo 